\title{
Design and Application of English Grammar Intelligent Question Bank System
}

\author{
Xiaowei Liu ${ }^{1}{ }^{1}$ and Hongjin Liu ${ }^{2}$ \\ ${ }^{1}$ School of Culture and Tourism, Wuxi Vocational College of Science and Technology, Wuxi 214000, China \\ ${ }^{2}$ Wuxi Hengding Supercomputing Center Co. Ltd, Wuxi 214000, China \\ Correspondence should be addressed to Xiaowei Liu; 3101015@wxsc.edu.cn
}

Received 30 August 2021; Accepted 19 October 2021; Published 29 October 2021

Academic Editor: Rahman Ali

Copyright (c) 2021 Xiaowei Liu and Hongjin Liu. This is an open access article distributed under the Creative Commons Attribution License, which permits unrestricted use, distribution, and reproduction in any medium, provided the original work is properly cited.

\begin{abstract}
The English grammar question bank can reserve a large number of special knowledge points and special categories of questions, which is helpful for English learners. Therefore, we design and verify the application effect of an intelligent question bank system. We construct an English grammar knowledge map, which is used to design the system architecture, including basic layer, data layer, service layer, business layer, and user layer. On the basis of the system structure, the index system of the item bank is established, and the functional modules of the system are designed comprehensively. Finally, we design a system database based on MySQL and give the table of knowledge points of English grammar. In order to verify the performance of the designed English grammar intelligent test bank system, a verification experiment is carried out. The experiment selects the accuracy rate of English grammatical feature extraction, recall rate, convergence, and system configuration accuracy. The experimental results show that the proposed system can extract English grammatical features with higher accuracy, and the recall rate is more satisfactory. Compared with the reference systems, the designed system configuration accuracy is higher.
\end{abstract}

\section{Introduction}

With the development of multimedia technology [1] and network communication technology [2], network-based distance learning has been paid more and more attention. It has the incomparable merit of the traditional teaching mode, creates a new teaching mode, breaks the limitation of the traditional teaching mode in time and space, adopts advanced teaching means and methods, improves teaching efficiency and teaching effect greatly, and makes teaching activities go to a new stage. The question bank system is a new type of CAI (computer-assisted interpreting) tool, which is developing rapidly at present. It is the product of the combination of computer science and educational theory science. It is a new direction of the CAI system. Test bank is a collection of test questions for a certain subject, which is composed in a computer system according to certain educational research theory. Under this background, with the development of educational informationization 2.0, more and more English learners tend to practice English questions online [3]. Because grammar is the basis of learning English, and grammar is usually listed as the most important part of English practice in many kinds of English practice questions. However, many kinds of grammatical problems are not intelligently processed in the current online learning platform.

Towards the grammar knowledge, English grammar study needs to pay attention to the grammar knowledge structure, therefore to the grammar knowledge spot demonstration, and needs to explore a clearer direct-viewing way. For the item bank system, it is of great importance to store a large number of topics of special knowledge points, which is helpful for the training of special topics. Second, it is also necessary to make a personalized learning path for students, which can reduce the waste of learning resources. In addition, teacher's resources are limited, the question bank system should support the knowledge spot content question and answer and real-time answers for the student 
to solve the question. To sum up, this paper will design and implement the English grammar intelligent test bank system, hoping to promote the development of the English grammar intelligent test bank system.

\section{Related Works}

Tong et al. [4] aims to realize a data-driven automatic prediction model of mathematical test difficulty by using test text and answer record data, and proposes a method based on the convolutional neural network $(\mathrm{CNN})$ and recurrent neural network, respectively, RNN's mathematical item difficulty prediction models c-midp (CNN for mathematical item difficulty prediction) and r-midp (RNN for mathematical item difficulty prediction), and their hybrid model h-midp (hybrid model for mathematical item difficulty prediction). Specifically, the proposed model is used to directly learn the text representation of test questions, and the score rate of test questions is used as the label training model. The whole process does not need to provide educational prior information such as knowledge labeling. Wei et al. [5] designs a distributed online examination system based on the SaaS mode. The question bank management module in the core business of the system constructs an examination question bank with its own attributes. After students $\log$ in to the system, they select examination subjects in the examination module. The question bank management module uses the intelligent test paper generation algorithm based on the improved genetic algorithm in the question bank according to the subjects selected by students, and then returns to the examinee page. After the examination, students and teachers can query the results in the information query and statistics module. Alghamdi et al. [6] proposed a two-phase intelligent examination system that consists of course management module and question and answer management module in which fuzzy logic-based methods are applied to get the question bank and its evaluation framework. They use the support vector machine algorithm to implement the AI-based classification system, which assign suitable questions to the respective students. $\mathrm{Hu}$ et al. [7] proposed an intelligent question bank system for medical students to improve their learning and research capabilities. Their system consists of a diversified, multidimensional, and hierarchical knowledge system of health statistics that consists of learning and examination materials. Their Internet-based health statistics intelligent education platform makes use of artificial intelligence technology to generate automatic tests.

However, after analysis, the English grammar test bank system in our country is still in the exploration stage of the intelligent learning platform, there are mainly the following problems:

(1) The presentation of knowledge points is illogical and not clear enough,

(2) The subject training in the item bank system does not support online answering, only the traditional questions are put on the website;
(3) The item bank system fails to obtain the user's record of questions to be made, and thus fails to recommend suitable topic training for the user,

(4) The Q\&A system of the item bank is not real-time. Although some websites provide answer functions, the time limit for answer is too slow, which affects the learning efficiency.

\section{Design of English Grammar Intelligent Question Bank System}

The performance of an item bank system [8] depends on the quality of the item bank and efficient management based on the item bank. There are at least two aspects of a high-quality item bank: one is whether the item bank can reasonably cover the content and knowledge of the course, and the other is whether the index system is scientific and reasonable. In addition, it is also very important whether the test paper generating system is reasonable or not, and whether the system has intelligent and learning functions. Of course, the number of questions in the library is also a very important factor, and it will directly affect the success or failure of the system.

3.1. Construction of English Grammar Knowledge Map. Combined with the characteristics of the English grammar knowledge map, this study constructs English grammar knowledge map [9]. The construction process can be divided into necessity analysis, English grammar data acquisition, knowledge map design, knowledge storage, and knowledge visualization [10] as shown in Figure 1.

(i) Step 1: Necessity analysis: In the first step, we analyze the business scenarios from four aspects of visualization, diversification, high efficiency, and depth whether it is necessary to construct Atlases [11]. The same information is expressed in images more vividly and intuitively than in words. Images present the grammatical knowledge structure system to users in a visual form and helps users to analyze the relationships and differences between grammatical knowledge points. Therefore, it is necessary to use knowledge Atlas to present English grammar knowledge points. Knowledge of English grammar includes the names, concepts, examples, and structures of grammar, and its data are diverse, not just a single type of data. Thus, the data on English grammar are diverse, and it is necessary to store them in knowledge maps [12].

(ii) Step 2: Get English grammar data from authoritative grammar books and Baidu Encyclopedia, including grammar names, concepts, examples, structures, etc. Table 1 lists the syntactic data sources and related explanations.

(iii) Step 3: Knowledge Atlas design, that is, the conceptual model design of Atlas, the main design of knowledge Atlas entity, and relationship. 


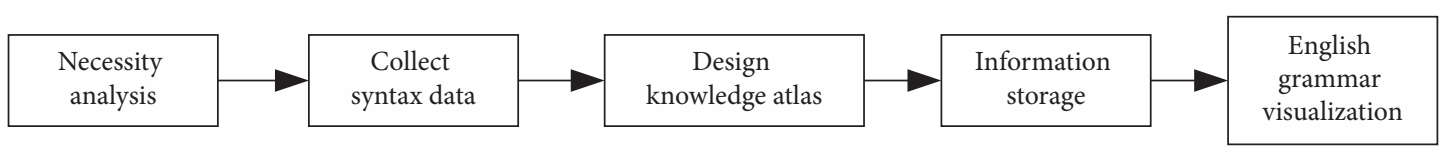

FIGURE 1: Steps of constructing English grammar knowledge map.

TABLe 1: Sources and descriptions of syntactic data.

\begin{tabular}{|c|c|c|}
\hline ar content source & Book name & Content obtained from books \\
\hline \multirow[b]{2}{*}{ English grammar books Baidu Encyclopedia } & FLTRP English grammar & $1.11=1$ \\
\hline & Modern English grammar & $\begin{array}{c}\text { The combination and structure of English grammar knowledge } \\
\text { points }\end{array}$ \\
\hline English grammar content source & English grammar entries & Concepts of English grammar and some examples \\
\hline
\end{tabular}

(a) Names of knowledge points of the word category. For example, nouns, adjectives, adverbs, verbs, and other parts of speech.

(b) The concept of word knowledge points. The concept of part of speech knowledge points refers to the concept of part of speech grammar. The concept of numerals, for example, refers to the number or order of words.

(c) Knowledge points of parts of speech examples of vocabulary. Part of speech knowledge point example vocabulary is referring to the part of speech grammar word example. Examples of adjectives include "great", “wide”, “clever", "happy", etc.

(d) Syntactic knowledge structure vocabulary. The syntactic knowledge point structure vocabulary refers to the syntactic knowledge point of the sentence structure-related vocabulary. For example, if the sentence structure of the simple future tense is "subject will + verb prototype", then "subject", "will", and "verb prototype" are the structural words of the simple future tense. Table 2 shows the categories of entity designs and their descriptions.

(iv) Step 4: Knowledge storage: Fill the data into the conceptual model of knowledge Atlas to form a complete knowledge Atlas

(v) Step 5: Knowledge visualization: Graphically present the knowledge Atlas you build

The knowledge Atlas constructed through the above contents can show the relationship and difference between the knowledge points of English grammar, which provides a database for English grammar intelligent question bank system.

3.2. System Architecture Design. The whole system is developed based on the five-tier architecture of the foundation layer, data layer, service layer, business layer, and user layer. According to the different structure and function of each layer, the system architecture designed in this paper is shown in Figure 2.
3.2.1. Basic Layer. The basic layer is the basic guarantee of the system construction, which includes the Internet construction and computer storage hardware construction $[11,12]$. Through the comprehensive infrastructure construction, it lays a good foundation for the overall construction of the whole item bank system.

3.2.2. Data Layer. It is of great importance to make an effective plan for the data layer because the system will display the data of English grammar knowledge comprehensively and realize a large number of topic resources. On the whole, the data resources of the item bank system are divided into Neo4j graph database resources [13] and MySQL relational database resources. The Neo4j graph database stores data of English grammar knowledge points [14], and MySQL stores data of the system's functionality.

3.2.3. Service Tier. The service layer is the main supporting technology of the system. The main technologies used in this paper are Flask web framework, Vue. js front-end framework, Cypher query, NLTK participle, and Jieba participle, etc. In order to realize the function of topic classification and intelligent question answering, we need feature extraction algorithms such as Word Bag, TF-IDF, Doc2vec, SVM, KNN, Naive Bayes, and so on. In addition, constructing knowledge Atlas and defining question templates are also very necessary technical support [15].

3.2.4. Business Layer. The business layer refers to the functions supported by the system. The system mainly supports four functions: subject classification, knowledge Atlas viewing, subject recommendation, and intelligent question answering. Topic classification can classify the grammar topics uploaded by users; knowledge map view supports users to view knowledge map as a whole and view according to conditions; topic recommendation is to recommend grammar topics for users, and users can answer online and get the answer results in a timely manner; intelligent question answering supports users to ask questions on grammar topics and return answers in a real-time manner [16]. 
TABLE 2: Knowledge Atlas design and explanation.

\begin{tabular}{lccc}
\hline Number & Category & Explanation & Example \\
\hline 1 & Concept of part of speech knowledge points & Concepts of English grammar & Proper nouns, especially words, etc. \\
2 & Name of part of speech knowledge points & Name of English grammar & Nouns, pronouns, and numerals \\
3 & Examples of parts of speech knowledge points & Examples of English grammar & Alice, Africa, rose \\
4 & Structure of part of speech knowledge points & The structure of English grammar & Is, going, to \\
\hline
\end{tabular}

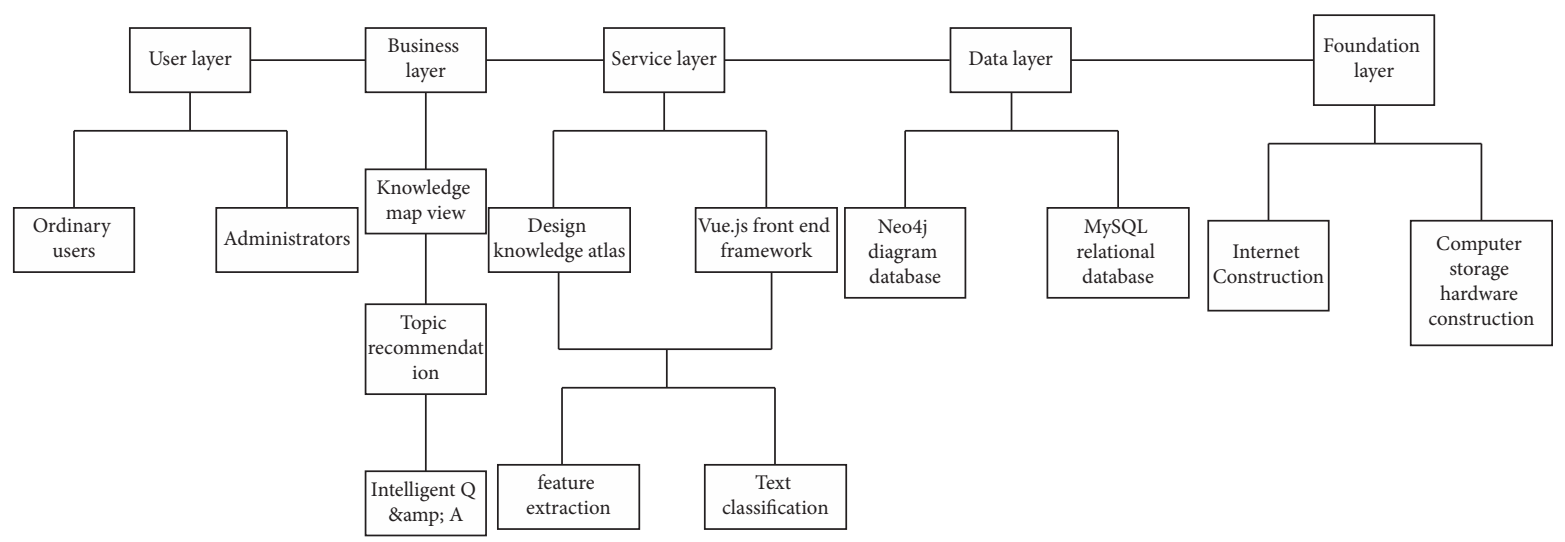

FIgURE 2: System architecture.

3.2.5. User Layer. The user layer refers to the objects used by the system. The users of the system can be divided into two categories: ordinary users and administrators. A common user can use knowledge Atlas view, title recommendation, and intelligent question answering function. An administrator can use title classification function more on this foundation [17].

3.3. Item Bank Index System. The index of the item bank is the description of the intrinsic attribute and external characteristic of the item bank and its function in the test. Whether the index system of the item bank and the specific index value of each item bank are appropriate or is not the key to reflect the high quality of item bank. Table 3 shows the item bank index system set by this system (the structure of item bank database).

Among them, in addition to the question bank itself and the reference answers, the meanings of the relevant index parameters are G1, G2, G3: the corresponding position of the content and knowledge points reflected in the question bank in the textbook (or syllabus); F: the score of the question bank; $\mathrm{T}$ : reference time for answers; $\mathrm{D}$ : difficulty coefficient of question bank; L: the type of question bank; Z: key item bank mark and random selection of the preferred object; $N$ : mark the interval times of the selected item bank, so as to ensure that the item bank is not selected repeatedly in consecutive examination papers. The practice shows that the system of the index value and the index value need to be quantified with examination theory, statistics theory, and expert experience to ensure the quality of paper in the future [18].

3.4. System Function Module. This system is mainly composed of several functional modules, such as item bank management, item card management, and item bank generation, as shown in Figure 3.
3.4.1. Question Bank Management Module and Examination Paper Bank Management Module. These two modules can realize the omnidirectional management of input, inquiry, maintenance, and printing. Among them, the examination papers stored in the database is the previous use of the paper and can be accessed at will, choice, for demonstration, or reuse.

3.4.2. Examination proposition Card Management Module. A test card is a form of test requirements filled out by the examiner, as shown in Table 4.

It is a frequently used way to automatically compose test paper by using the requirement of a test paper proposition card. The test paper cards can also be archived, retained, and used for all-round management.

3.4.3. Examination Paper Generation Module. This module provides an expert model, test paper proposition card model, and manual model of the three ways of paper selection.

Expert mode test paper. Using the expert's knowledge and experience, comprehensive and standardized requirements are put forward, and such requirements are preset in the system; once the function is selected, you can automatically get a number of papers. Such an examination paper should be able to embody the characteristics of knowledge of the course, with authority. This module also provides an update and improve the function of the expert mode.

Test paper proposition card mode test paper. The form of the paper proposition card (see Table 2) filled out by the examinee shall be put into the system, and the system will automatically select the questions from the examination question bank as required. The characteristic of the model is 
TABLE 3: Database structure of the item bank.

\begin{tabular}{ccccccccccc}
\hline & & Chapter & Section & Paragraph & Score & Time & Difficulty & Type & Key & Frequency \\
\hline Test questions & Answer & G1 & G2 & G3 & F & T & D & L & Z & N \\
\hline
\end{tabular}

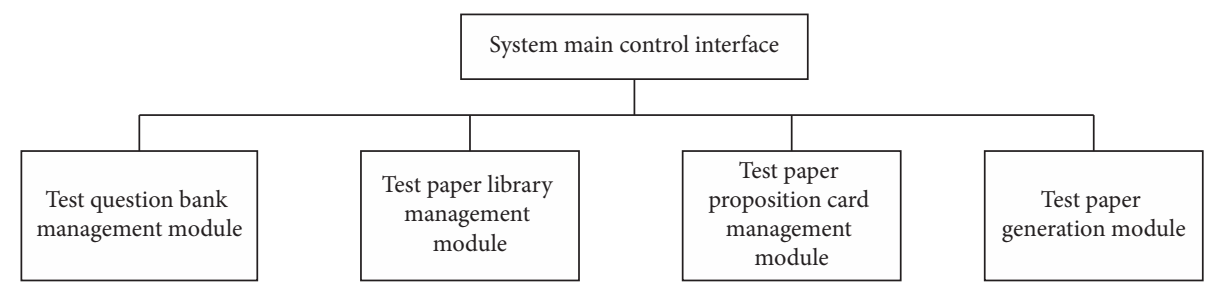

Figure 3: System function module diagram.

that the same card table can produce several papers with the same specifications and requirements, which can be used as $\mathrm{A}$ and $\mathrm{B}$ papers or as a whole.

Hand generating paper. Using this model, the system will allow you to calmly browse the entire database of related attribute indicators, and the operator can easily browse while selecting the test bank to form a paper. The above three methods can be chosen according to the actual situation, thus the system provides users with a variety of flexible means of paper.

3.4.4. Functional Module of Subject Classification Based on Knowledge Atlas. There are four processes in this paper: obtaining training data, text preprocessing, feature vector design by extracting knowledge map features and parts of speech features of correct answers, and constructing an SVM classifier $[13,14]$. The activity diagram is shown in Figure 4 . The acquisition of topic training data refers to the acquisition of English grammar topic data. In this paper, a certain number of English grammar topics are obtained from the English Test Question Bank. English grammar knowledge points usually appear in the first major category of examination papers in the form of single-choice questions. In order to adapt to the general form of English grammar questions, this paper selects English grammar single-choice questions. Therefore, the topic data obtained in this paper includes question stem, choice, correct choice, answer analysis, and so on. The activity diagram of topic classification based on knowledge map is shown in Figure 4.

Text preprocessing refers to the preprocessing of the subject data obtained to facilitate the direct use of the data in subsequent steps. Preprocessing includes standardized processing, word segmentation, and part-of-speech tagging. Standardization refers to filling up the missing parts in the question stem with the correct answers, namely, getting the correct answers and the complete English grammar sentences with the correct answers. Word segmentation refers to the use of the NLTK word segmentation tool to cut complete English grammar sentences into individual words. Part of speech tagging refers to the use of NLTK word segmentation tools to mark the parts of speech of each word, such as proper nouns, adjectives, verbs, and so on. The feature vector design is to extract numerical features from title text data and convert the text into the vector form to facilitate the use of subsequent training classifiers. The eigenvectors in this paper include the eigenvectors based on knowledge Atlas, the eigenvectors based on part of speech of correct answer, and the eigenvectors based on TF-IDF. The feature vectors based on knowledge Atlas are mainly obtained from knowledge Atlas according to entity return relation, and the feature vectors based on the correct part of speech are mainly obtained from word segmentation results of preprocessing steps, and the feature vectors based on TFIDF are mainly obtained from complete English grammar sentences.

\subsubsection{Design of Intelligent Question Answering Function} Module Based on Knowledge Map. The intelligent question answering based on knowledge Atlas is mainly divided into three processes: question classification, template matching, and answer generation. Its activity diagram is shown in Figure 5.

Question classification is to match the natural language question input by the user to the number of the predefined question and the corresponding question template through the question classifier. Question classification is also a process of text classification in essence, so we need to design a classifier. Question classification can be divided into five steps: designing question template, preparing template training dataset, text preprocessing, feature vector design, and constructing classifier.

3.4.6. Modification Module. Users may modify part of their own information (including user account, password, and e-mail, etc.), and administrators may modify administrator information and modify the examination question information.

If you want to modify the test information, in the query results show the page, click the question below the "modify" button, and you can go to the test modification page. Figure 6 shows the flow chart of the system algorithm for question modification.

An example of the SQL statements used to modify the database is the following: 
TABLE 4: Test paper proposition card.

\begin{tabular}{|c|c|c|c|c|c|c|c|}
\hline \multicolumn{8}{|c|}{$\begin{array}{l}\text { English grammar test card } \\
\text { Course name }\end{array}$} \\
\hline Serial number & Chapter & Section & Paragraph & Fraction & Time & Difficulty & Type \\
\hline & G & $\mathrm{R}$ & $\mathrm{U}$ & $\mathrm{F}$ & $\mathrm{T}$ & $\mathrm{N}$ & $\mathrm{M}$ \\
\hline \multicolumn{8}{|l|}{1} \\
\hline \multicolumn{8}{|l|}{2} \\
\hline \multicolumn{8}{|l|}{3} \\
\hline \multicolumn{8}{|l|}{4} \\
\hline \multicolumn{8}{|l|}{5} \\
\hline Total & & & & 100 points & 100 minutes & & \\
\hline
\end{tabular}

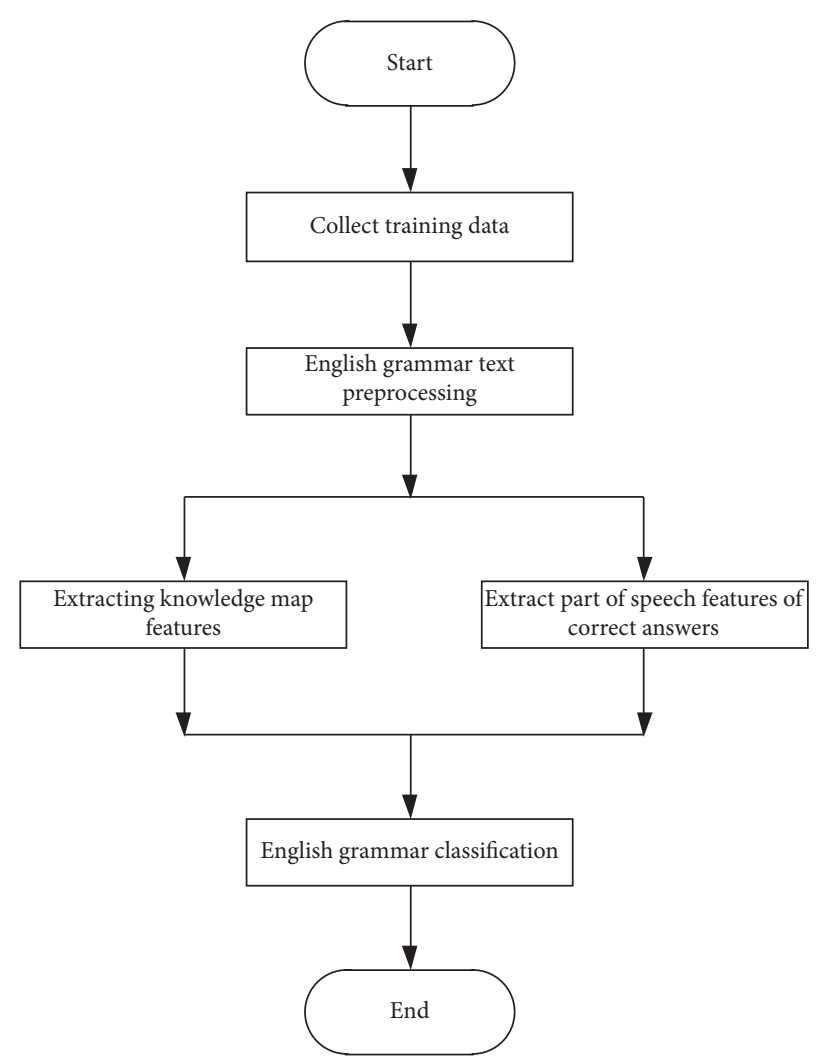

FIgURE 4: Activity diagram of topic classification based on knowledge map.

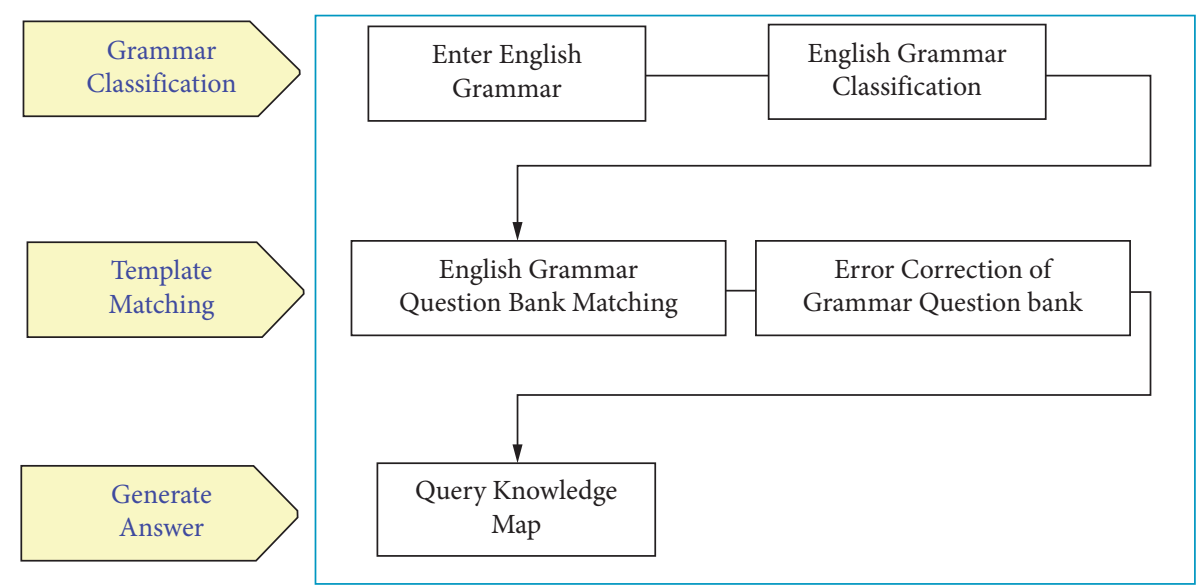

FIGURE 5: Intelligent Q\&A, a diagram based on knowledge map. 


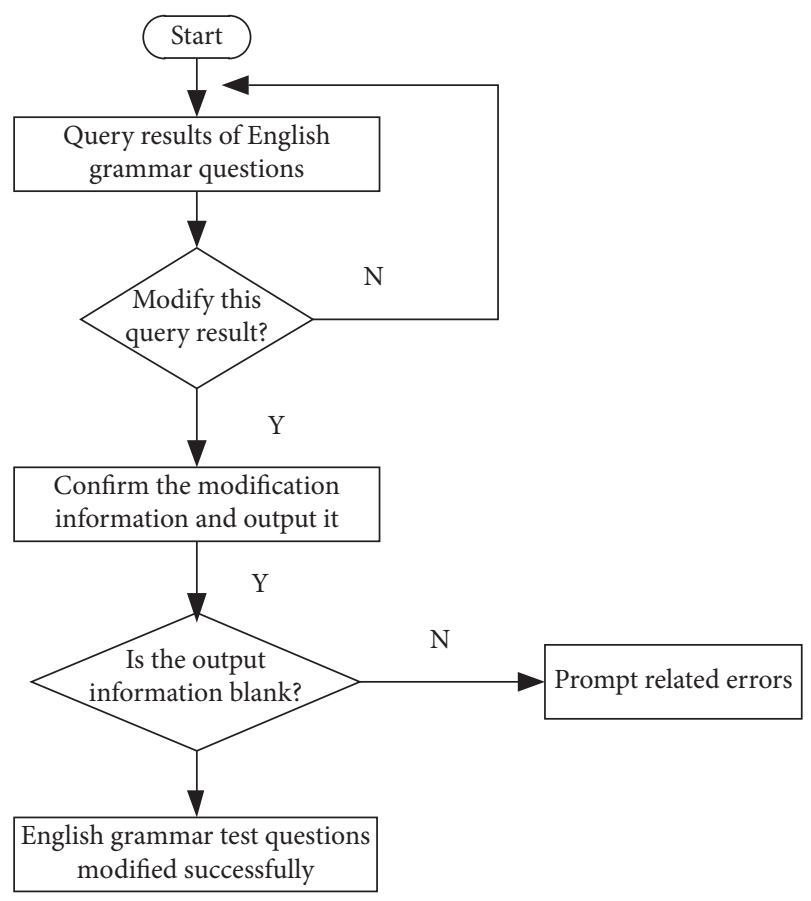

Figure 6: Algorithm flow chart of test question modification system.

SqlString = "update test set $\operatorname{diff}=$ "“+diff+", point $=$ "“+point+"”, title $=$ "“+title+"”, answer_ $a=$ "“+answer_ $a+$ "”, answer_b = " + answer_ $b+$ +", $\quad$ answer_c $=$ "“+answer_c+”, answer_d $d=$ “"+answer_ $d+$ "”, rightanswer = “"+rightanswer+"”, type $=$ “"type+" "where count $=$ "+count;

3.5. Design of System Database Based on MySQL. MySQL is an open source relational database system. MySQL is one of the best relational database management system applications in the web application. Therefore, the system uses MySQL to manage the data of the English grammar test bank system. In actual application scenario, common entity has a knowledge dot, title, user, and make a problem to record.

The data information of knowledge points mainly includes the serial number, name, and category of knowledge points; the data information of topics mainly includes the content of the question stem, optional content, correct option, answer analysis, title number, and knowledge point number; the data information table of users mainly includes the serial number, name, password, and other user information; the inscription table mainly includes the serial number, user number, title number, user answer, right or wrong answer, and time of question making. The title number and the knowledge point number of each topic are unique, thus facilitating the call to the topic. There is a corresponding relationship between knowledge points and topics: a topic corresponds to a knowledge point, and a knowledge point corresponds to a number of topics; there is a correlation between a topic and a record of doing questions: a record of doing questions corresponds to a topic; there is a relationship between a user and a record of doing questions: a user corresponds to a record of doing questions, a record of doing questions corresponds to a user. Table 5 shows the structure of one of the database tables, i.e., the knowledge point information table.

\section{Application Effect Verification of English Grammar Intelligent Question Bank System}

In order to verify the performance of the designed English grammar intelligent test bank system, the following experiments are designed. The experiment selects the accuracy rate of English grammatical feature extraction, recall rate, convergence, and system configuration accuracy. The specific contents of the experiment are given below.

First, the accuracy of feature extraction is compared. The index is expressed as the ratio of correct features in the extracted feature as follows:

$$
\text { precision }=\frac{n^{\prime}}{n}
$$

where $n^{\prime}$ represents the number of correct features, also known as the number of hits, and $n$ is the total number of subject words. The experimental comparison results of the two systems are shown in Figure 7.

Second, the recall rate of the two systems is analyzed. The recall rate is also called the recall rate. The calculation formula is as follows:

$$
\text { recall }=\frac{n^{\prime}}{N}
$$

Comparison of feature extraction recall rates of different systems is shown in Figure 8.

From the above two pictures, we can see that the recall rate of the system is higher than that of the competitor system, and the recall rate is similar to the accuracy of the competitor system.

Based on the distribution of resources statistics shown in Figure 9, this paper analyzes the features of English grammar intelligent question bank, builds the model of English grammar intelligent question bank sharing information mining and feature clustering analysis, and extracts the features of English grammar intelligent question bank under the network environment through the statistical average analysis system. The results of the convergence curve shared by English grammar intelligent question bank is shown in Figure 9.

Analysis of Figure 9 shows that the system achieves the lowest convergence error of 0.02 in 4 min when the initial convergence error is the same. The convergence error of the system in [4] reaches the lowest value of 0.11 at $9 \mathrm{~min}$, and in [5], it reaches the lowest value of 0.17 at $10.5 \mathrm{~min}$. The above experimental data show that the proposed system has good convergence in scheduling English grammar intelligent test questions under the network environment.

The convergence curve of learning information scheduling of English grammar question bank becomes flat after some time, and as a result, the errors tend to zero. The rationale behind flattening is that the proposed method uses SQL-based indexing method as compared to its counterparts, which 
TABLE 5: Knowledge point information table.

\begin{tabular}{lccc}
\hline Field name & Type & Attribute & Description \\
\hline KNOWLEDGE-vocabulary & INTEGER & NOT NULL & Types of knowledge points \\
KNOWLEDGE-semantics & TEXT & NOT NULL & Contents of knowledge points \\
KNOWLEDGE-ID & TEXT & NOT NULL & Location of knowledge points \\
KNOWLEDGE-name & TEXT & PRIMARY KEY & Naming of knowledge points \\
\hline
\end{tabular}

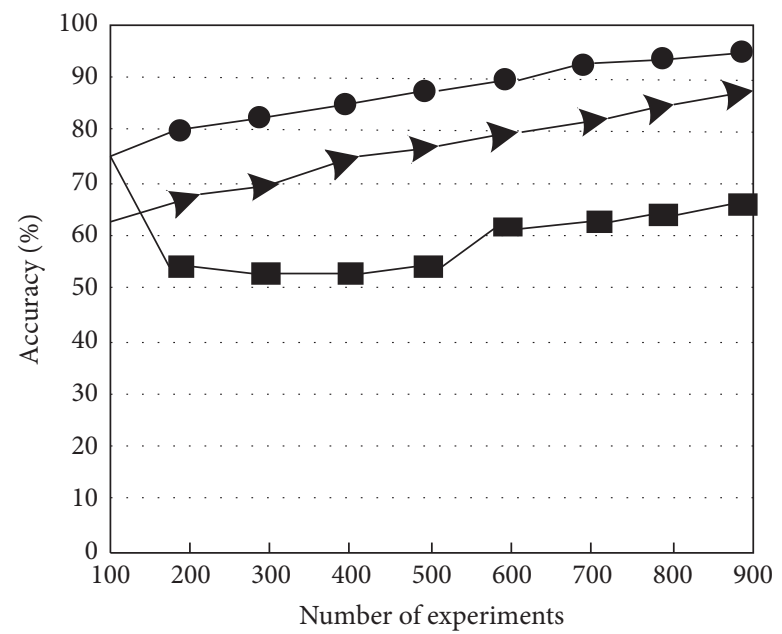

The system designed in this paper

System designed in reference [4]

System designed in reference [5]

Figure 7: Comparison of feature extraction accuracy of different systems.

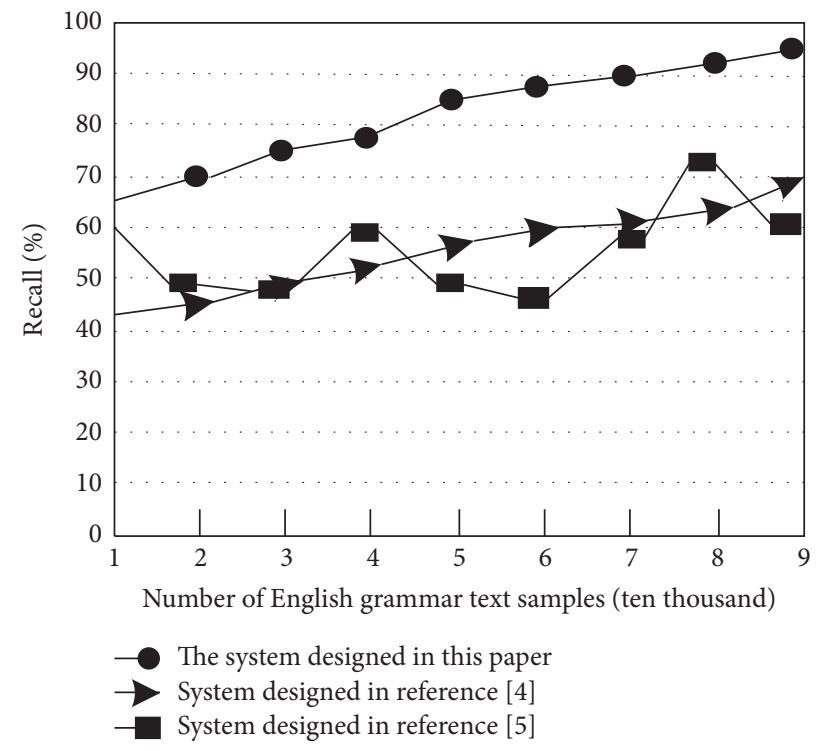

Figure 8: Comparison of feature extraction recall rates of different systems.

provides a method for sorting contents based on pointers. The data are physically located on data stores. This improves the searching performance and reduce the missing ratio, which ultimately reduce the error rate. It is just like the index in the back of a massive book, which makes it faster to find something quickly and accurately without missing the content. 


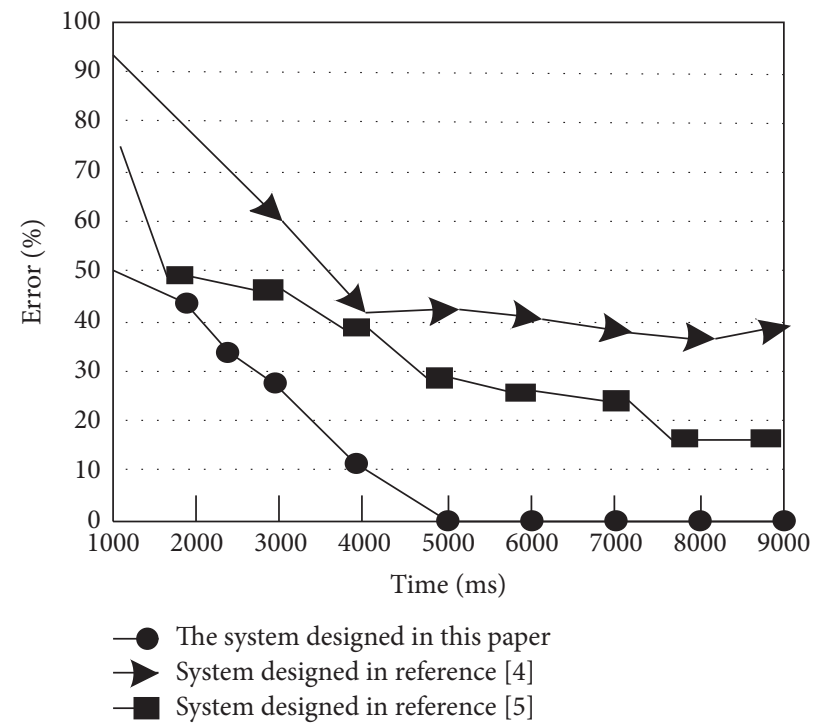

FIgURE 9: Convergence curve of learning information scheduling of English grammar question bank.

TABLE 6: Comparison of collocation accuracy in English grammar question bank.

\begin{tabular}{lccc}
\hline Number of iterations & Proposed system & System designed in reference [4] & System designed in reference [5] \\
\hline 1000 & 0.981 & 0.812 & 0.833 \\
2000 & 0.992 & 0.835 & 0.816 \\
3000 & 0.961 & 0.800 & 0.791 \\
4000 & 0.934 & 0.813 & 0.761 \\
5000 & 0.984 & 0.841 & 0.732 \\
6000 & 0.986 & 0.854 & 0.710 \\
7000 & 0.995 & 0.704 & 0.699 \\
8000 & 0.995 & 0.696 & 0.672 \\
9000 & 0.928 & 0.653 & 0.655 \\
10000 & 0.919 & 0.527 & 0.642 \\
\hline
\end{tabular}

We tested the accuracy of English grammar question bank configuration under different systems. The results are shown in Table 6.

As shown in Table 1, in the calculation process of multiple iterations, the average accuracy of our proposed system reaches 0.978 , while the average accuracy of the system in reference [4] and reference [5] are 0.812 and 0.724 , respectively, which are lower than that of the proposed system.

\section{Conclusion}

In order to enhance the special learning effect of English grammar, an intelligent question bank system of English grammar is designed. Knowledge Atlas is introduced to design the overall architecture of the system. Based on this, the index system of item bank is established, and the functional modules of the system are designed. MySQL is used to design the system database. The experimental results verify that the accuracy, recall, and system configuration accuracy of the designed system are ideal, which shows that the system can provide a reliable basis for intelligent learning of English grammar.

\section{Data Availability}

The data used to support the findings of this study are available from the corresponding author upon request.

\section{Conflicts of Interest}

The author declares that there are no conflicts of interest regarding this study.

\section{Acknowledgments}

This paper did not receive any financial support.

\section{References}

[1] K. Scheiter, C. Schubert, A. Schüler et al., "Adaptive multimedia: using gaze-contingent instructional guidance to provide personalized processing support," Computers \& Education, vol. 139, pp. 31-47, 2019.

[2] Z. Xu, J. Lu, X. Wang, J. Zhang, M. Alazab, and V. G. Díaz, “AI and machine learning for the analysis of data flow characteristics in industrial network communication security," International Journal of Ad Hoc and Ubiquitous Computing, vol. 37, no. 3, pp. 125-136, 2021. 
[3] J. Anthony, "The English language learning progressions (ELLP): challenge or opportunity?" Curriculum Matters, vol. 16, pp. 21-37, 2020.

[4] W. Tong, "Data driven prediction for the difficulty of mathematical items," Journal of Computer Research and Development, vol. 56, no. 5, p. 1007, 2019.

[5] Z. Q. Wei, Y. Yuan, and J. Cai, "Design of distributed online examination system based on SaaS mode," Modern Electronics Technique, vol. 42, no. 12, pp. 48-51, 2019.

[6] A. A. Alghamdi, M. A. Alanezi, and F. Khan, "Design and implementation of a computer aided intelligent examination system," International Journal of Emerging Technologies in Learning (iJET), vol. 15, no. 1, pp. 30-44, 2020.

[7] H. Hu, J. Li, X. Lei, P. Qin, and Q. Chen, "Design of health statistics intelligent education system based on Internet+," Journal of Physics: Conference Series, IOP Publishing, vol. 1168, no. 6, 2019.

[8] A. O. Flentje, I. Caturegli, and S. M. Kavic, "Practice makes perfect: introducing a question bank for ABSITE preparation improves program performance," Journal of Surgical Education, vol. 77, no. 1, pp. 54-60, 2020.

[9] A. Lackner, S. Fathalla, M. Nayyeri et al., "Analysing the evolution of computer science events leveraging a scholarly knowledge graph: a scientometrics study of top-ranked events in the past decade," Scientometrics, vol. 126, no. 9, pp. 8129-8151, 2021.

[10] M. Yu, T. Quan, Q. Peng, X. Yu, and L. Liu, "A model-based collaborate filtering algorithm based on stacked AutoEncoder. Neural Computing and Applications," Neural Computing \& Applications, vol. 4, 2021.

[11] Y. M. Chen and C. Y. Li, "Simulation of Target Tactical intention recognition based on knowledge Map," Computer Simulation, vol. 36, no. 8, pp. 1-4, 2019.

[12] F. Long, "Simulation of English text recognition model based on ant colony algorithm and genetic algorithm," Journal of Intelligent \& Fuzzy Systems Preprint, vol. 2, pp. 1-12, 2021.

[13] Z. He, "English grammar error detection using recurrent neural networks," Scientific Programming, vol. 2021, pp. 1-8, 2021.

[14] L. H. Vonti and A. Rosyid, "The effectiveness OF vocabulary games IN English grammar class,” Pedagonal: Jurnal Ilmiah Pendidikan, vol. 3, no. 2, pp. 43-55, 2019.

[15] X. Yu, J. Yang, and Z. Xie, "Training SVMs on a bound vectors set based on Fisher projection," Frontiers of Computer Science, vol. 8, no. 5, pp. 793-806, 2014.

[16] C.-F. Wu, Y.-H. Chang, M.-C. Yang, and T.-W. Kuo, "When storage response time catches up with overall context switch overhead, what is next?" IEEE Transactions on ComputerAided Design of Integrated Circuits and Systems, vol. 39, no. 11, pp. 4266-4277, 2020.

[17] X. Yu, Y. Chu, F. Jiang, Y. Guo, and D. Gong, "SVMs classification based two-side cross domain collaborative filtering by inferring intrinsic user and item features," KnowledgeBased Systems, vol. 141, pp. 80-91, 2018.

[18] S. Li, Q. Zhang, X. Xin et al., "No prior recognition method of modulation mode by partition-fractal and SVM learning method," Chinese Optics Letters, vol. 18, no. 11, Article ID 111404, 2020. 